\title{
Extraction of Image Parking Spaces in Intelligent Video Surveillance Systems
}

\author{
Rykhard Bohush ${ }^{1}$, Pavel Yarashevich ${ }^{1}$, Sergey Ablameyko ${ }^{2}$, Tatiana Kalganova ${ }^{3}$ \\ 1 Polotsk State University, Polotsk, Belarus \\ 2 Belarusian State University, Minsk, Belarus \\ ${ }^{3}$ Brunel University, London, UK \\ r.bogush@psu.by
}

\begin{abstract}
This paper discusses the algorithmic framework for image parking lot localization and classification for the video intelligent parking system. Perspective transformation, adaptive Otsu's binarization, mathematical morphology operations, representation of horizontal lines as vectors, creating and filtering vertical lines, and parking space coordinates determination are used for the localization of parking spaces in a video frame. The algorithm for classification of parking spaces is based on the Histogram of Oriented Descriptors (HOG) and the Support Vector Machine (SVM) classifier. Parking lot descriptors are extracted based on HOG. The overall algorithmic framework consists of the following steps: vertical and horizontal gradient calculation for the image of the parking lot, gradient module vector and orientation calculation, power gradient accumulation in accordance with cell orientations, blocking of cells, second norm calculations, and normalization of cell orientation in blocks. The parameters of the descriptor have been optimized experimentally. The results demonstrate the improved classification accuracy over the class of similar algorithms and the proposed framework performs the best among the algorithms proposed earlier to solve the parking recognition problem.
\end{abstract}

Key words: parking space, localization, Histogram of Oriented Descriptors, classification, Support Vector Machine.

\section{Introduction}

The importance of video surveillance systems become more and more important in different types of human activity. The development in computer vision technologies made it possible thanks for recent development of video surveillance systems with intelligent processing of input video data for various applications, including car parking control systems.

Over the last decade the numerous intelligent systems have been developed for parking space detection with different lighting conditions and restrictions $1,2,6,14,24,25$. For example, a system for management and monitoring of a parking lot by a video camera for simple indoor parking garage proposed in $\sqrt{6}$ uses the edge detection method for identification of the free parking places within indoor scenario only with a permanent lightning source. Parking system described in the paper 25] uses the brown rounded 
image drawn at parking spaces and produces the information of empty spaces. A display shows the number of currently available parking lots. This system was tested and results were presented only for 8 parking spaces. A video-based system for vacant parking space detection based on colour histograms and difference of Gaussian features, SVM classifier, and exponential smoothing for temporal integration is presented in 24]. This system can be adopted by a car-park routing system to navigate drivers to a comfortable parking space. Authors proposed to use Raspberry Pi in a video camera in 2]. That work is based on deep Convolution Neural Network (CNN) architecture to classify images of parking spaces as occupied or vacant and exhibits a very high accuracy. However, such approach is computationally expensive.

The systems like those just described can be integrated in a large system known as Smart City and some optional functionality for both parking owners and parking users can be implemented [1,2,18. For example, for parking owner, an important additional information is the number of automobiles in the parking lot, the automobile types, the human activity. Car owners need background information about vacant parking space location, car surveillance with a smartphone, alarming via smartphone in case of an extraordinary situation [16. In such systems the advanced, efficient video data processing algorithms are necessary, which need large computational resources. They should operate in the presence of various noise factors: shadows, light spots in sunny weather, changes in the overall illumination of the automotive parking during the day, changing weather conditions, etc.

The methods that determine the occupancy of parking spaces on video images can be divided into three groups. The first group includes methods based on detection of a car in a parking lot [5,22, the second group contains methods based on a comparison of the processed parking space with the reference vacant space [20] and the third group includes from combined methods 12,15.

The methods from the first group are unstable due to classification errors, which arise because of the overlap of cars in video images. The second group of methods are based on the model of reference vacant parking space, so the probability of false classification increases with the appearance of natural noise, the presence of people and other objects in an image, the local change in illumination, etc. In the combined methods, as a rule, the positive properties of the component methods are emphasized and their negative sides are avoided, but computing cost of these methods are usually large.

Among the features to be chosen to form vectors of image characteristics, HOG [7] is a good candidate. This is due to that it has a number of desirable characteristics, like the invariance to image rotation and scale changing, and the stability to noise and illumination variations. Recently, a range of methods and algorithms using HOG gradients have been proposed $[10,11,12,13]$. In the method [11 the HOG descriptor and Bayesian classifier was used, and the correct classification probability was 0.9945 when the images were taken in typical lighting conditions. In this method it is assumed that 
a structural 3D model of each parking place is built, so the computation time is long. The method described in 12 is more effective than those previously mentioned and attains an increased probability of correct classification in case of less controlled shooting conditions. An improvement of this algorithm by introducing the SVM classification method is presented in [13. As a result, the probability of correct classification became 0.9955. In [10], the parking lots classification algorithm based on HOG and SVM is compared with the algorithm based on Haar-like features and AdaBoost method. For the first algorithm, the probability of correct classification is equal to 0.691, while for the second method this probability is 0.95. Hence, despite of good characteristics of the both methods the problem which is our object of interest has not being solved yet. Therefore, this area of research is still of great interest.

This paper is organized as follows. In Section 2, an intelligent parking lot inspection system, suitable for scaling and having some optional service functionalities is illustrated. In Section 3, the algorithm for locating the parking spaces in the parking lot image and the algorithm for space classification are presented. In Section 4, experimental results of the search for more effective descriptor parameters and for better class separating functions in the classifier are performed. The correct classification probability of parking spaces of the present algorithm is compared to those of the other approaches. Finally, a conclusion is provided in Section 5 .

\section{Intelligent Parking Lot Control System}

Intelligent parking lot control system is shown in Fig. 1. The system consists of video camera, video processing module, service functions module, information representation device or display and cloud platform for integration with the Smart City. Video information processing module includes subsystems for parking space localization and subsystems for parking space classification.

Parking space localization subsystem is intended to locate parking spaces in video frame and save these positions in Local Storage. This is required when the point of view is changing after the installation of a new system or camera. Input image perspective transformation is used to facilitate possibility of describing parking space by rectangle, to detect separating parking lines and should be found once for a given car park and camera location. For parking space segmentation is mandatory a specialized processing algorithm. In the last step, coordinates of parking spaces are saved in local storage. This data will be used by subsystem of parking spaces classification.

Parking space classification subsystem captures a video, processes it frame-by-frame and present the result for the current frame. This subsystem is used to dedicate following contributions: coordinates of parking space extraction from local storage; feature extraction for each image of parking space; parking spaces classification to "vacant" and "occupied" based on features; merging results from the previous step with input video 


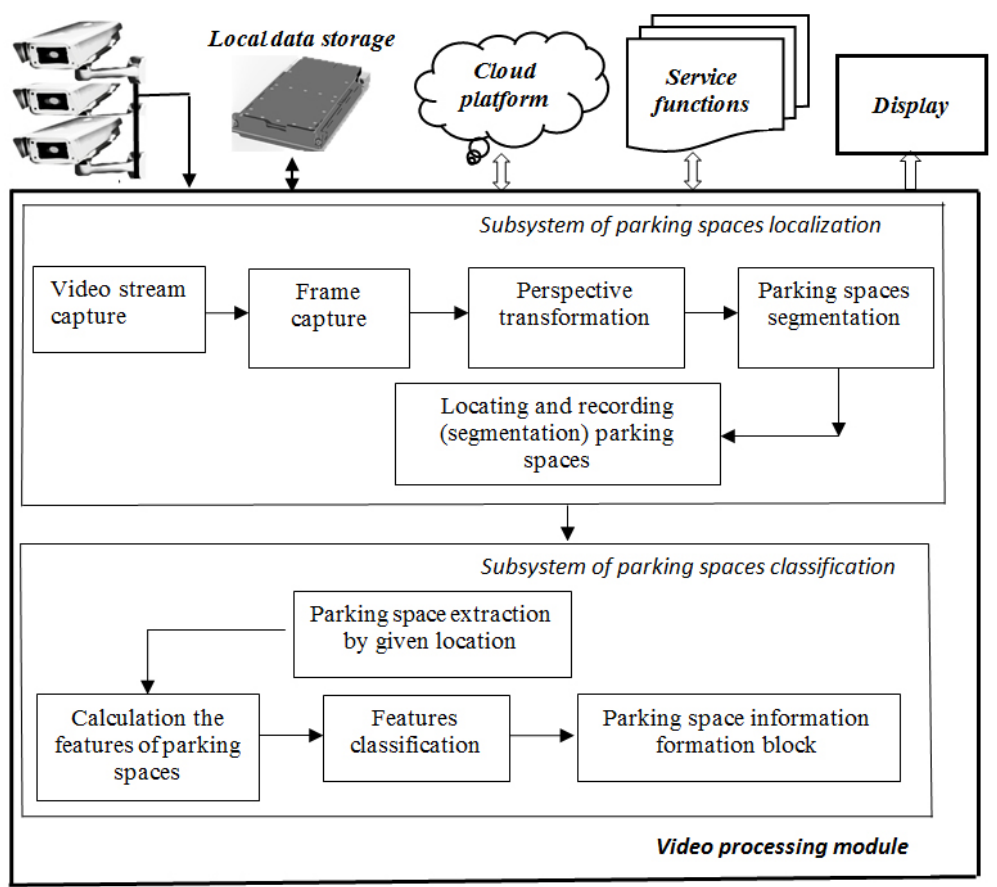

Fig. 1. The structure of the intelligent parking lot control system.

frame for visualization on a display of occupied and vacant parking spaces. System capability can be escalated by optional module "Service functions". This module can consist of car counter on parking lot, car types recognition, map of vacant parking spots, car surveillance with smart phone, etc. This approach can help to solve an important problem of huge negative environmental impact by minimizing it. The level of car emissions will significantly decrease, as a driver can quickly find a vacant parking spot in a large parking lot.

\section{Parking space extraction algorithms}

\subsection{Parking spaces localization}

The system determine locations of parking spaces when a new system is installed or the camera's point of view is changed. Parking space location is describes by four coordinates in image of parking lot. Locations of Parking spaces stored in Local Storage. Algorithm of parking space localization includes the following steps: 
1. Perspective transformation of parking lot image. The perspective transformation is: $(x, y, 1)=H \times(x, y, 1)^{T}$, where $(x, y, 1)$ are homogeneous pixel coordinates in the image of parking lot, $\left(x^{\prime}, y^{\prime}, 1\right)$ are corresponding homogeneous pixel coordinates in the output image, $H$ - homography matrix. The pixels coordinates $\left(x_{p}, y_{p}\right), p=\overline{1,4}$ on the image of parking lot and corresponding pixels coordinates $\left(x_{p}^{\prime}, y_{p}^{\prime}\right), p=\overline{1,4}$ on the output image are used to compute homography matrix. Pixels coordinates $\left(x_{p}, y_{p}\right)$ are defined manually, when perspective transformation is applying the first time. These coordinates are the corners of the closest parking row. Pixels coordinates $\left(x_{p}, y_{p}^{\prime}\right)$ describe a rectangular area. The output image after perspective transformation is larger than the original image. Now, each parking space represents a rectangle area, and its sides are parallel to the axes.

2. Otsu method is used to binarize the image.

3. A closing operation of mathematical morphology is used to remove gaps on the lines that separate parking spaces from each other:

$$
A \bullet B=(A \oplus B) \ominus B
$$

where $(A \oplus B)$ is dilation of image $A$ by the structuring element $B$ of $7 \times 7$ and $\ominus$ is erosion. An opening operation of mathematical morphology is used to shrink areas of small noise and unrelated elements:

$$
A \circ C=(A \ominus C) \oplus C
$$

where $C$ - the structuring element of $5 \times 5$.

4. Each inseparable horizontal sequence of pixels is represented by horizontal line $V_{i}\left(x_{i}^{, h l}, y_{i}^{, h l}, l_{i}\right), i=\overline{0, n o v-1}$, where $\left(x_{i}^{, h l}, y_{i}^{, h l}\right)$ is a start pixel of horizontal line, $l$ is a length of horizontal line, nov is a total number of lines. The one pixel represents as $\left(x^{, h l}, y^{, h l}, 1\right)$.

5. Horizontal lines $V_{i}\left(x_{i}^{, h l}, y_{i}^{, h l}, l_{i}\right)$ are stacking into vertical lines $L_{j}(\bar{V}), j=\overline{0, n o l-1}$, nol - total number of vertical lines. Vectors form vertical line and they should be one under another and should not exceed neighboring vectors by length. They stacked into vertical line $L_{j}\left(x_{j}^{, v l}, y_{j}^{, v l}, w_{j}^{, v l}, h_{j}^{, v l}\right)$, where $\left(x_{j}^{, v l}, y_{j}^{, v l},\right)$ is a start pixel of vertical line, $w_{j}, h_{j}$ are width and height of vertical line. Each vertical line $L_{j}$ covers every horizontal line over a rectangle area $x_{j}^{, v l}, y_{j}^{, v l}, x_{j}^{, v l}+w_{j}^{, v l}, y_{j}^{, v l}+h_{j}^{, v l}$.

6. The lines are sorted according to the initial coordinates: from the left to the right, from the top to the bottom. Then they are combined into parking spaces $S_{k}\left(L_{k}^{l}, L_{k}^{r}\right)$, $k=\overline{0, n o s-1}$, where $L_{k}^{l}, L_{k}^{r}$ are the left and the right dividing strips respectively, nos is a number of parking spaces.

7. Parking spaces $S_{k}\left(x_{k}^{, s}, y_{k}^{, s}, w_{k}^{, s}, h_{k}^{, s}\right), k=\overline{0, n o s-1}$ are described by its initial coordinates $\left(x^{, s}, y^{, s}\right)$, its width $w^{, s}$ and height $h^{, s}$. 


\subsection{Feature set computation}

1. The subimage of parking space $I\left(w^{, s}, h^{, s}\right)$ is extracted according to its location $S\left(x^{s}, y^{s}, w^{, s}, h^{, s}\right)$. Then it is scaled into subimage $I(w, h)$, where $(w, h)$ is the size of the subimage.

2. Magnitude of gradient $G$ and orientation of gradient $\theta$ is calculated for grayscaled subimage of parking space $I$ as:

$$
\begin{aligned}
G_{x, y} & =\sqrt{{G_{x, y}^{x}{ }^{2}+G_{x, y}^{y}{ }^{2}}^{x}} \\
\theta_{x, y} & =\arctan \left(\frac{G_{x, y}^{x}}{G_{x, y}^{y}}\right)
\end{aligned}
$$

where $G_{x, y}^{x}$ and $G_{x, y}^{y}$ - horizontal and vertical gradients:

$$
\begin{aligned}
& G_{x, y}^{x}=I_{x, y} \cdot M^{x} \\
& G_{x, y}^{y}=I_{x, y} \cdot M^{y}
\end{aligned}
$$

where $0 \leq x<w, 0 \leq y<h, M^{x}$ and $M^{x}$ - derivative kernels $\left(M^{x}=M^{y T}=\right.$ $[-1,0,+1])$.

3. Magnitude of gradients $G$ is divided into cells $C_{m, n}$ with size $C_{w} \times C_{h}$ and power gradient accumulation in accordance with orientations $\theta$ for each cell:

$$
C_{m, n}^{l}=\sum_{\substack{j=n \cdot c h \\
i=m \cdot c w}}^{\substack{(n+1) \cdot c h-1 \\
(m+1) \cdot c w-1}}\left\{\begin{array}{l}
G_{i, j}, \frac{l \cdot 2 \cdot \pi}{b} \leq \theta_{i, j}<\frac{(l+1) \cdot 2 \cdot \pi}{b} \\
0, \frac{l \cdot 2 \cdot \pi}{b}>\theta_{i, j} \bigcup \theta_{i, j} \geq \frac{(l+1) \cdot 2 \cdot \pi}{b}
\end{array}\right.
$$

where $b$ - a number of orientation bins in cell, $0 \leq l<b, 0 \leq m<c w, 0 \leq n<c h$, $c w=\frac{w}{C_{w}}, c h=\frac{h}{C_{h}}$.

4. Cells are united into overlapping blocks $B_{f, g}$ with size $B_{w} \times B_{h}$ :

$$
B_{f, g}=\left[\begin{array}{ccc}
C_{f, g} & \cdots & C_{f+B_{w}-1, g} \\
\vdots & \ddots & \vdots \\
C_{f, g+B_{h}-1} & \cdots & C_{f+B_{w}-1, g+B_{h}-1}
\end{array}\right]
$$

where $0 \leq m<b w, 0 \leq g<b h, b w=c w-B_{w}+1, b h=c h-B_{h}+1$. 
5. The L2-norm block normalization scheme is used. Some cells present in several overlapping blocks, and each of them normalized with respect to the block it belongs to:

$$
C_{i, j}^{l}{ }^{f, g}=\frac{C_{i, j}^{l}}{\left\|B_{f, g}\right\|_{2}}=\frac{C_{i, j}^{l}}{\sqrt{\sum_{i=f}^{f+B_{w}-1} \sum_{j=g}^{g+B_{h}-1} \sum_{l=0}^{b-1} C_{i, j}^{l}{ }^{2}+\epsilon^{2}}}
$$

where $\epsilon$ is a small constant, prevents from division by zero.

6. Normalized blocks are sequentially added to the descriptor. Values of cells $C_{i, j}^{l}{ }^{f, g}$ in blocks $B_{f, g}$ are grouped together to construct a feature vector:

$$
d_{b \cdot B_{w} \cdot B_{h} \cdot(b w \cdot g+f)+b \cdot\left(B_{w} \cdot(j-g)+(i-f)\right)+l}=C_{i, j}^{l}{ }^{f, g}
$$

Vector size of $d$ depends on size $w \times h$ of parking space subimage, size of cell $C_{w} \times C_{h}$, numbers of orientations in cell $b$ and size of block $B_{w} \times B_{h}$ :

$$
D_{S}=b \cdot B_{w} \cdot B_{h} \cdot\left(\frac{w}{C_{w}}-1\right) \cdot\left(\frac{h}{C_{h}}-1\right)
$$

\subsection{Feature set classification}

We use support vector machine to classify computed descriptors of parking space [21]. Support Vector Machine is a linear classifier. To perform a non-linear classification kernel trick is used. Support Vector Machine is used for binary classification to distinguish vacant parking spaces from occupied:

$$
a(d)=\operatorname{sign}\left(\sum_{i=0}^{n} \lambda_{i} y_{i} K\left(d_{i}, d\right)-w_{0}\right)
$$

where $a(d)$ - returns +1 if parking space is vacant, and -1 if parking space is occupied; $\lambda\left(\lambda_{0}, \lambda_{1}, \ldots, \lambda_{n}\right)$ - a vector of dual variables; $d_{i}, i=1, n$ - descriptors of support vectors; $y\left(y_{0}, y_{1}, \ldots, y_{n}\right)$ - an array of class labels; $w_{0}$ - a threshold value; $d$ - a descriptor of parking space subimage to be classified. Classification efficiency depends on kernel functions. For parking lot classification, the following kernel functions can be used:

- linear:

$$
K_{\operatorname{lin}}\left(d_{a}, d_{b}\right)=d_{a} \cdot d_{b}
$$

where $d_{a}$ and $d_{b}$ - descriptors.

- radial basis function:

$$
K_{\mathrm{rbf}}\left(d_{a}, d_{b}\right)=\exp \left(-\gamma \cdot\left\|d_{a}-d_{b}\right\|^{2}\right)
$$


- polynomial:

$$
K_{\mathrm{poly}}\left(d_{a}, d_{b}\right)=\left(\gamma \cdot K\left(d_{a}, d_{b}\right)+c\right)^{\delta}
$$

$\gamma, \delta, c-$ parameters of corresponding kernel functions.

- histogram intersection:

$$
K_{\text {hist }}\left(d_{a}, d_{b}\right)=\sum \min \left(\left|d_{a i}\right|,\left|d_{b i}\right|\right)
$$

To achieve good results of classification the parameters of Histogram of Oriented Gradients and Support Vector Machine should be chosen attentively. Relevant experiments are described in the next section.

\section{Experimental results and discussion}

\subsection{Evaluation metrics definition}

For our experiments, we used the PKLot dataset 8 . PKLot dataset contains images of parking lot taken in different weather conditions, shadow effects and illumination. Pictures from this dataset taken by a camera, that is installed high enough and located in the middle of the right parking row, and rows behind it. So the perspective transformation could be applied to receive the top view of parking lot image, without overlapping between vehicles.

Experiments on described algorithms and evaluation of research result was realized using programming language Java, Eclipse development environment, computer vision libraries OpenCV 3.0.0 and machine learning library jlibsvm.

Fig. 2 shows parking lot image (Fig. 2a) and results for some steps (Fig. 2p-f) of parking space localization algorithm. Parking lot image after perspective transformation, binarization and applying morphological operations is presented in Fig. 2b. Fig. $2 \mathrm{~F}$ is a subimage from Fig. $2 \mathrm{~b}$. Fig. $2 \mathrm{~d}$ shows a result after horizontal and vertical lines definition on the subimage (Fig. 22). Results parking spases localization for subimage and all parking lot are presented in Fig. 2p and Fig. 2f correspondingly. We use 2135 images of parking lot from PKLot dataset. The total number of subimages of parking spaces is 292808. Fig. 3 shows some examples for vacant and occupied parking spaces. Main characteristics of parking images for different weather conditions are presented in Tab. 1. Descriptor efficiency is evaluated by correctly parking space images classification probability $R R$ that can be represented as:

$$
R R=\frac{T P+T N}{T P+F P+T N+F N}
$$

where $T P$ - correctly classified vacant parking spaces; $F P$ - incorrectly classified vacant 

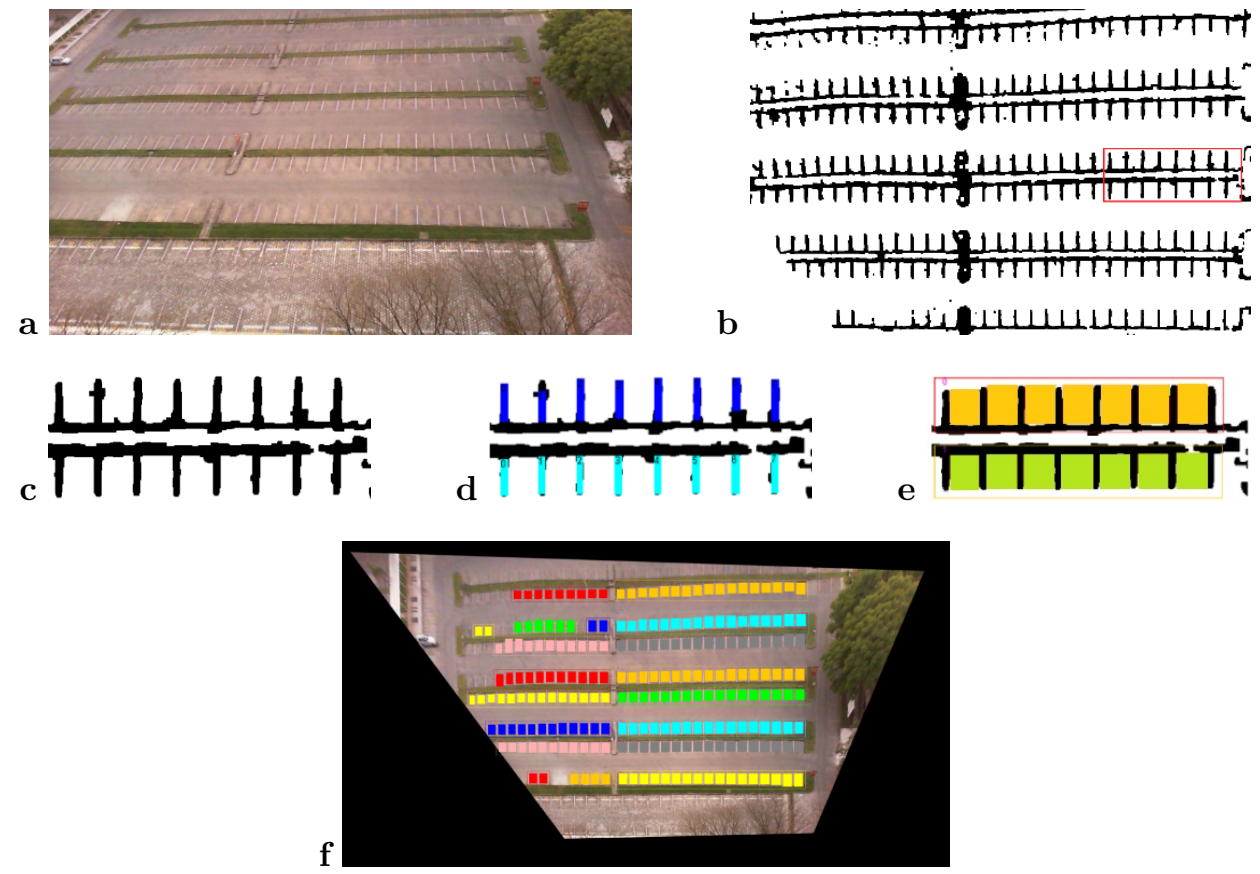

Fig. 2. Example of parking spaces localization algorithm. (a) The image of parking lot; (b) the result of applying the first, the second and the third step; (c) the subimage of Fig. 2b; (d) the result of applying the fourth and the fifth step; (e) after applying the sixth and the seventh step; (f) localized parking spaces.

Tab. 1. Main characteristics of parking spaces subimages.

\begin{tabular}{|c|c|c|c|}
\hline \multirow{2}{*}{$\begin{array}{c}\text { Weather } \\
\text { conditions }\end{array}$} & \multirow{2}{*}{$\begin{array}{c}\text { Number of days/ } \\
\text { number of images }\end{array}$} & \multicolumn{2}{|c|}{$\begin{array}{c}\text { Number of subimages } \\
\text { of parking spaces }\end{array}$} \\
\cline { 3 - 4 } & & vacant & occupied \\
\hline Mist & $6 / 664$ & 61406 & 28898 \\
\hline Rain & $4 / 416$ & 18670 & 37906 \\
\hline Shiny & $12 / 1073$ & 78801 & 67127 \\
\hline
\end{tabular}

parking spaces; $T N$ - correctly classified occupied parking spaces; $F N$ - incorrectly classified occupied parking spaces. The probability of falsely vacant parking space subimage classification is calculated as follows:

$$
F P R=\frac{F P}{T P+F P}
$$



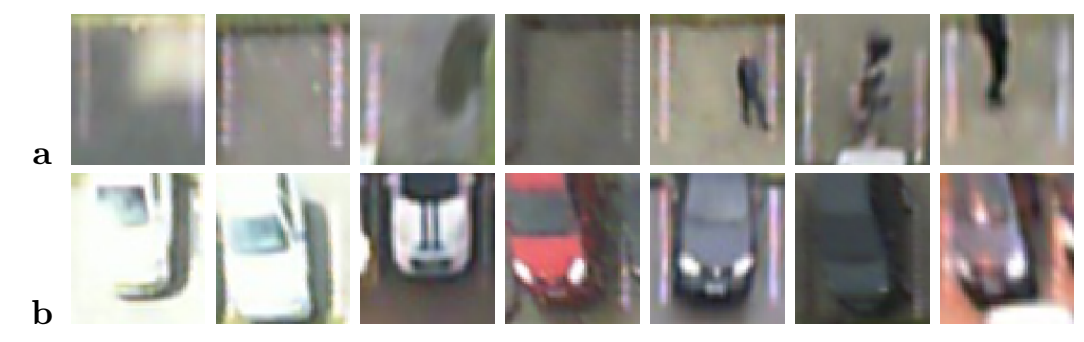

Fig. 3. Examples for image of parking spaces: (a) vacant; (b) occupied.

The probability of falsely occupied parking space subimage classification is defined as equation:

$$
F N R=\frac{F N}{T N+F N}
$$

\subsection{Experiments and its results}

Proposed model for parking lot classification is consists of HOG descriptor and SVM classifier. Parameters of descriptor and classifier should be optimized to receive high efficiency model. HOG includes a large number of parameters to optimize: the size of parking space subimage, the size of cell, the number of orientation bins, the size of block. It is very hard problem to find an optimal set for all the possible combinations of parameters. Therefor, fixed sequence of parameters would be used to optimize: $w, h, C_{w}, C_{h}, b$, where $C_{w}, C_{h}$ are width and height of cell; $b$ is number of orientations in cell. At the first step, dual-dimension parameters (the size of parking space subimage, the size of cell) would be determined. After that, the one-dimensional parameter (number of orientation cells) should be determined. At each step, parameters will be selected to reduce the descriptor size. This approach would reduce computational cost for parking space classification subsystem.

The highest probability of correct classification was achieved by using the following sizes of parking space subimage: $64 \times 56,64 \times 64,64 \times 72,64 \times 80,48 \times 104$. We used $8 \times 8$ cell and 9 bins cell histogram, which are effective for other practical tasks, for example, pedestrian detection The experimental results are shown in Fig. 4 . Figures 4-6 are the heatmap diagrams. Such a diagram represents a value by a color, a minimum and maximum values are represented by white and black colors correspondingly. White rectangles show parameter values that are selected for next step. Next, highest probability of correct classification was achieved for parking spaces subimage size and cells size $\left(w, h, C_{w}, C_{h}\right):(64,56,8,8),(64,64,8,8),(64,72,8,6),(64,72$, 8, 8), (64, 72, 8, 9), (64, 72, 8, 12), (64, 80, 8, 5), (64, 80, 8, 8), (64, 80, 8, 16), (48, $104,8,8)$. The experimental results are shown in Fig. 5. At last step, we find the 


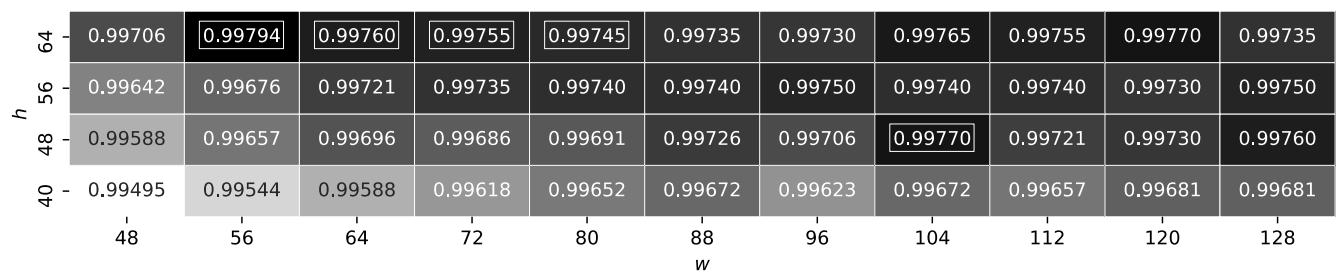

Fig. 4. Probability of correct classification for different size of parking space subimage.
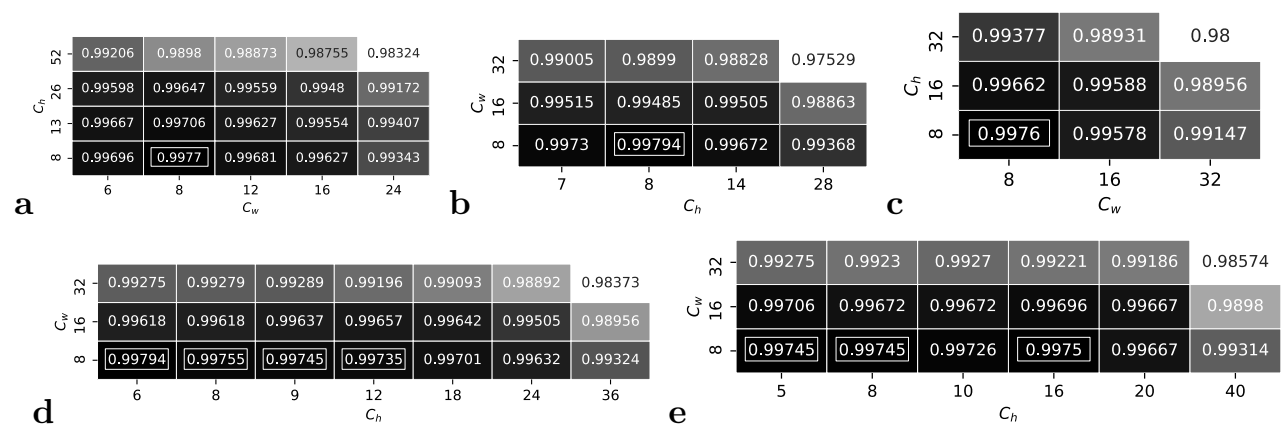

Fig. 5. Correct classification probability for different cell's size with size of parking space subimage. (a) $64 \times 56$; (b) $64 \times 64 ;$ (c) $64 \times 72 ;$ (d) $64 \times 80 ;$ (e) $48 \times 104$.

orientation numbers in cells that provide maximal correct detection probability on the interval from 4 to 18 bins histogram. Experimental results for the number of orientations in the cell showed that for the interval from 4 to 8 bin, values of right detecting probability are not acceptable. Therefore, experimental results from 9 to 18 are shown in fig. 6. The highest probability of correct classification was achieved by using for following parameters $\left(w, h, C_{w}, C_{h}, b\right):(64,72,8,6,14),(64,72,8,6,16),(64,72,8$, $8,16)$. The feature set length of obtained descriptors are $D_{s}(64,72,8,6,14)=4928$, $D_{s}(64,72,8,6,16)=5638, D_{s}(64,72,8,8,16)=3584$. So, the most effective descriptor given reduce computation cost has the following parameters: parking space size is $64 \times 72$, cell size is $8 \times 8$, and orientations number in cell is 16 .

SVM classification result efficiency is depends on the type of kernel function. Therefore, experiments were conducted to evaluate the effectiveness of SVM classification of parking space characteristics based on HOG using various kernel functions Tab.2 (where KF - kernel function, SSW - separating strips wide, SVN - support vector number, Lin. - linear kernel, H. I. - histogram intersection kernel, R. B. F. - Radial Basis Function, Poly - polynomial kernel). The polynomial kernel shows the highest $R R$, but we choose 


\begin{tabular}{|c|c|c|c|c|c|c|c|c|c|}
\hline$(64,80,8,8)$ & 0.99711 & 0.99775 & 0.99686 & 0.99789 & 0.99799 & 0.99775 & 0.99789 & 0.99799 & 0.99789 \\
\hline$(64,80,8,5)-$ & 0.99706 & 0.99735 & 0.99672 & 0.99765 & 0.99789 & 0.99779 & 0.99789 & 0.99784 & 0.99784 \\
\hline$(64,80,8,16)-$ & 0.99716 & 0.99755 & 0.99681 & 0.99745 & 0.9977 & 0.9974 & 0.99721 & 0.99726 & 0.99765 \\
\hline$(64,72,8,9)-$ & 0.99735 & 0.99765 & 0.99672 & 0.99765 & 0.99789 & 0.99765 & 0.99794 & 0.99784 & 0.99794 \\
\hline$(64,72,8,8)$ & 0.99726 & 0.99779 & 0.99676 & 0.99775 & 0.99804 & 0.99775 & 0.99819 & 0.99809 & 0.99789 \\
\hline$(64,72,8,6)$ & 0.99701 & 0.99779 & 0.99681 & 0.99789 & 0.99819 & 0.99799 & 0.99819 & 0.99804 & 0.99814 \\
\hline$(64,72,8,12)$ & 0.99745 & 0.99765 & 0.99681 & 0.9973 & 0.99794 & 0.99716 & 0.99779 & 0.99755 & 0.99794 \\
\hline$(64,65,8,8)-$ & 0.99701 & 0.99814 & 0.99613 & 0.99799 & 0.99789 & 0.99765 & 0.9977 & 0.99794 & 0.99799 \\
\hline$(64,64,8,8)$ & 0.99721 & 0.99779 & 0.99681 & 0.9976 & 0.99789 & 0.99745 & 0.99784 & 0.99779 & 0.99804 \\
\hline$(48,104,8,8)-$ & 0.99726 & 0.9975 & 0.99623 & 0.99735 & 0.9976 & 0.99686 & 0.99711 & 0.9973 & 0.99765 \\
\hline & 10 & 11 & 12 & 13 & $\begin{array}{c}14 \\
b\end{array}$ & 15 & 16 & 17 & 18 \\
\hline
\end{tabular}

Fig. 6. Probability of correct classification for different orientations numbers in cells: $(64,56,8,8),(64,64,8,8)(64,72,8,6),(64,72,8,8),(64,72,8,9),(64,72,8,12)$, $(64,80,8,5),(64,80,8,8),(64,80,8,16),(48,104,8,8)$.

Tab. 2. Efficiency comparison of different kernel function.

\begin{tabular}{|l|c|r|c|c|c|}
\hline \multicolumn{1}{|c|}{ KF } & SSW & SVN & RR & FPR & FNR \\
\hline Lin. & 4.366 & 161 & 0.99431 & $2.91 \mathrm{e}-3$ & $9.16 \mathrm{e}-3$ \\
\hline H. I. & 2.387 & 262 & 0.99652 & $2.12 \mathrm{e}-3$ & $5.19 \mathrm{e}-3$ \\
\hline R. B. F. & 1.371 & 330 & 0.99706 & $1.41 \mathrm{e}-3$ & $4.86 \mathrm{e}-3$ \\
\hline Poly. & 0.752 & 479 & 0.99740 & $1.58 \mathrm{e}-3$ & $3.86 \mathrm{e}-3$ \\
\hline
\end{tabular}

histogram intersection kernel because it classifies much more faster then radial basic function kernel or polynomial kernel.

Fig. 7] shows the example of parking space classification using the developed algorithm with defined parameters under different weather conditions. Every image of parking space has been correctly classified. Comparison of the proposed algorithm with other classification of parking space image algorithms is shown in Tab. 3.

These results show that correct classification of image parking spaces based on proposed descriptor parameters is more attractive with other descriptor parameters.

Comparison of classification algorithms for PKLot dataset is presented in Tab. 4. As we can see in this table, the approach is the most effective among other approaches, which using PKLot dataset for testing. 

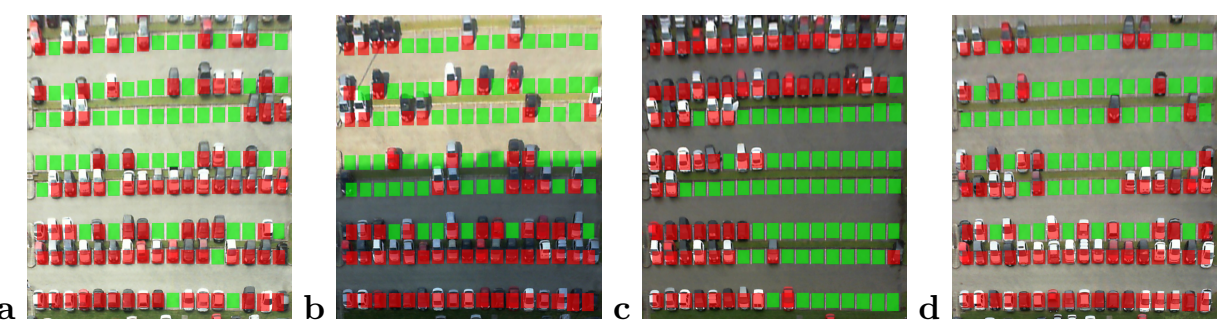

Fig. 7. The result of parking spaces classification under different weather conditions. (a) Sunny; (b) Shadow cast; (c) Rainy; (d) Cloudy.

Tab. 3. Characteristics comparison of classification algorithms for parking spaces.

\begin{tabular}{|c|c|c|c|c|c|}
\hline Algorithm & Descriptor & Classifier & $\begin{array}{l}\text { No. of } \\
\text { parking } \\
\text { lot } \\
\text { images }\end{array}$ & $\begin{array}{c}\text { No. of } \\
\text { parking } \\
\text { spaces }\end{array}$ & $\mathbf{R R}$ \\
\hline C. C. Huang 11 & HOG $(64,32,16,16,8)$ & Bayes & 955 & 72 & 0.9945 \\
\hline C. C. Huang 12 & HOG $(64,32,16,16,10)$ & Bayes & 825 & 72 & 0.9939 \\
\hline C. C. Huang 13 & HOG $(96,48,-,-,-)$ & SVM & 1,564 & 72 & 0.9955 \\
\hline R. Fusek 10 & HOG $(96,96,8,8,4)$ & SVM & - & 57 & 0.6910 \\
\hline R. Fusek 10 & Haar & Adaboots & - & 57 & 0.9500 \\
\hline \multirow{4}{*}{$\begin{array}{l}\text { M. } \\
\text { Tschentscher } \\
23\end{array}$} & Histogram, HSV & \multirow{2}{*}{$\mathrm{k}-\mathrm{NN}$} & \multirow{4}{*}{1,010} & \multirow{4}{*}{36} & 0.9655 \\
\hline & DoG & & & & 0.9358 \\
\hline & Histogram, RGB & & & & 0.9712 \\
\hline & DoG & $S \vee M$ & & & 0.9413 \\
\hline \multirow{3}{*}{ L. Baroffio 4} & \multirow{3}{*}{ Histogram, HSV } & \multirow{3}{*}{ SVM } & 3,791 & 28 & 0.9600 \\
\hline & & & 4,152 & 37 & 0.9300 \\
\hline & & & 4,474 & 100 & 0.8700 \\
\hline Proposed & $\operatorname{HOG}(64,72,8,8,16)$ & SVM & 2,153 & 136 & 0.9970 \\
\hline
\end{tabular}

Tab. 4. Comparison of classification algorithms for PKLot dataset.

\begin{tabular}{|c|c|c|c|}
\hline Author & Classifier & Descriptor & $\mathbf{R R}$ \\
\hline L. Baroffio et al. $\sqrt{4}$ & SVM & Color Hist. & 0.960 \\
\hline G. Amat et al. 3 & mAlexNet & - & 0.904 \\
\hline D. D. Mauro et al. $\overline{9}$ ] & mAlexNet & - & 0.990 \\
\hline G. Amato et al. 2 & mAlexNet & - & 0.996 \\
\hline X. Li et al. 17 & GAN & - & 0.957 \\
\hline S. Nurullayev et al. 19 & CarNet & - & 0.982 \\
\hline Proposed & SVM & $\mathrm{HOG}$ & 0.997 \\
\hline
\end{tabular}




\section{Conclusion}

The intelligent parking lot control system is proposed. It includes the video camera, the video processing module, the cloud platform, the service functions module, and the information representation device or display. In this system, Efficient algorithms for parking space localization and classification have been proposed. The algorithm for localization of parking spaces in the parking lot image consists of the following steps: perspective transformation, Otsu's binarization, operations of mathematical morphology, lines construction and coordinates of parking spaces determination. Histogram of Oriented Descriptors and Support Vector Machine are used to classify the parking spaces. The algorithm for HOG computation consists of the following steps: vertical and horizontal gradient calculation for image of parking lot, gradient module vector and orientation calculation, power gradient accumulation in accordance to the cell orientations, blocking of cells, second norm calculations, and normalization of cell orientation in blocks. The kernel function based on histogram intersection is the most effective to classify the computed HOG feature sets for car parking spaces. The most effective parameters of the descriptor were found to be as follows: $64 \times 72$ - the size of parking space subimage, $8 \times 8$ - the size of cell, 16 - the number of orientation bins. The correct classification rate is 0.997 . The experimental results demonstrate the improved classification accuracy.

\section{References}

[1] M. Alam, D. Moroni, G. Pieri, M. Tampucci, M. Gomes, J. Fonseca, J. Ferreira, and G. R. Leone. Real-time smart parking systems integration in distributed ITS for smart cities. Journal of Advanced Transportation, 2018, 2018. doi:10.1155/2018/1485652.

[2] G. Amato, F. Carrara, F. Falchi, C. Gennaro, C. Meghini, and C. Vairo. Deep learning for decentralized parking lot occupancy detection. Expert Systems with Applications, 72:327-334, 2017. doi:10.1016/j.eswa.2016.10.055

[3] G. Amato, F. Carrara, F. Falchi, C. Gennaro, and C. Vairo. Car parking occupancy detection using smart camera networks and deep learning. In Proc. IEEE Symp. on Computers and Communication (ISCC 2016), pages 1212-1217. IEEE, 2016. doi:10.1109/iscc.2016.7543901

[4] L. Baroffio, L. Bondi, M. Cesana, A. E. Redondi, and M. Tagliasacchi. A visual sensor network for parking lot occupancy detection in smart cities. In Proc. IEEE 2nd World Forum on Internet of Things (WF-IoT 2015), pages 745-750, 2015. doi:10.1109/WF-IoT.2015.7389147.

[5] D. B. L. Bong, K. C. Ting, and N. Rajaee. Car-park occupancy information system. In Proc. 3rd Real-Time Technology and Applications Symposium (RENTAS 2006), pages 65-70, Serdang, Selangor, Malaysia, 2006.

[6] T. Čaklović, I. Aleksi, and Ž. Hocenski. Managing and monitoring of a parking lot by a video camera. In Proc. of Automation in Transportation, Zagreb, Croatia, 2010. http://bib.irb.hr/ datoteka/509872. Automatizacija_u_prometu_2010.pdf

[7] N. Dalal and B. Triggs. Histograms of oriented gradients for human detection. In Proc. Int. Conf. on Computer Vision \& Pattern Recognition (CVPR'05), volume 1, pages 886-893. IEEE, 2005. doi:10.1109/CVPR.2005.177. 
[8] P. RL. De Almeida, L. S. Oliveira, A. S. Britto, E. J. Silva, and A. L. Koerich. PKLot - a robust dataset for parking lot classification. Expert Systems with Applications, 42(11):4937-4949, 2015. doi:10.1016/j.eswa.2015.02.009

[9] D. Di Mauro, S. Battiato, G. Patanè, M. Leotta, D. Maio, and G. M. Farinella. Learning approaches for parking lots classification. In Proc. Int. Conf. on Advanced Concepts for Intelligent Vision Systems (ACIVS 2016), volume 10016 of Lecture Notes in Computer Science, pages 410-418. Springer, 2016. doi:10.1007/978-3-319-48680-2_36

[10] R. Fusek, K. Mozdřeň, M. Šurkala, and E. Sojka. Adaboost for parking lot occupation detection. In Proc. 8th Int. Conf. on Computer Recognition Systems CORES 2013, volume 226 of Advances in Intelligent Systems and Computing, pages 681-690. Springer, 2013. doi:10.1007/978-3-319-00969-8_67

[11] C. C. Huang, Y. S. Dai, and S. J. Wang. A surface-based vacant space detection for an intelligent parking lot. In Proc. 12th Int. Conf. on ITS Telecommunications, pages 284-288. IEEE, 2012. doi:10.1109/ITST .2012.6425183

[12] C. C. Huang, Y. S. Tai, and S. J. Wang. Vacant parking space detection based on plane-based bayesian hierarchical framework. IEEE Transactions on Circuits and Systems for Video Technology, 23(9):1598-1610, 2013. doi:10.1109/TCSVT.2013.2254961

[13] C. C. Huang, H. T. Vu, and Y. R. Chen. A multiclass boosting approach for integrating weak classifiers in parking space detection. In Proc. 2015 IEEE Int. Conf. on Consumer Electronics Taiwan, pages 314-315. IEEE, 2015. doi:10.1109/ICCE-TW.2015.7216918

[14] M. Y. I. Idris, Y. Y. Leng, E. M. Tamil, N. M. Noor, and Z. Razak. Car park system a review of smart parking system and its technology. Information Technology Journal, 8(2):101-113, 2009. doi:10.3923/itj.2009.101.113

[15] J. Jermsurawong, M. U. Ahsan, A. Haidar, H. Dong, and N. Mavridis. Car parking vacancy detection and its application in 24-hour statistical analysis. In Proc. 10th Int. Conf. on Frontiers of Information Technology (FIT 2012), pages 84-90. IEEE, 2012. doi:10.1109/FIT.2012.24.

[16] J. Lanza, L. Sánchez, V. Gutiérrez, J. A. Galache, J. R. Santana, P. Sotres, and L. Muñoz. Smart city services over a future internet platform based on internet of things and cloud: The smart parking case. Energies, 9(9):719, 2016. doi:10.3390/en9090719

[17] X. Li, M. C. Chuah, and S. Bhattacharya. Uav assisted smart parking solution. In Proc. Int. Conf. on Unmanned Aircraft Systems (ICUAS 2017), pages 1006-1013. IEEE, 2017. doi:10.1109/icuas.2017.7991353

[18] R. Novotnỳ, R. Kuchta, and J. Kadlec. Smart city concept, applications and services. Journal of Telecommunications System \& Management, 3(2):1, 2014. doi:10.4172/2167-0919.1000117

[19] S. Nurullayev and S. W. Lee. Generalized parking occupancy analysis based on dilated convolutional neural network. Sensors, 19(2):277, 2019. doi:10.3390/s19020277

[20] R. J. L. Sastre, P. G. Jimenez, F. J. Acevedo, and S. M. Bascon. Computer algebra algorithms applied to computer vision in a parking management system. In Proc. IEEE Int. Symp. on Industrial Electronics (ISIE 2007), pages 1675-1680. IEEE, 2007. doi:10.1109/ISIE.2007.4374856

[21] M. O. Stitson, J. A. E. Weston, A. Gammerman, V. Vovk, and V. Vapnik. Theory of support vector machines. Technical Report CSD-TR-96-17, Department of Computer Science, Royal Holloway University of London, 1996.

[22] N. True. Vacant parking space detection in static images. Technical report, University of California, San Diego, 2007. http://cseweb.ucsd.edu/classes/wi07/cse190-a/reports/ntrue.pdf. 
[23] M. Tschentscher, C. Koch, M. König, J. Salmen, and M. Schlipsing. Scalable real-time parking lot classification: An evaluation of image features and supervised learning algorithms. In Proc. Int. Joint Conf. on Neural Networks (IJCNN 2015), pages 1-8. IEEE, 2015. doi:10.1109/IJCNN. 2015.7280319

[24] M. Tschentscher and M. Neuhausen. Video-based parking space detection. In Proc. Forum Bauinformatik, pages 159-166, 2012.

[25] R. Yusnita, F. Norbaya, and N. Basharuddin. Intelligent parking space detection system based on image processing. International Journal of Innovation, Management and Technology, 3(3):232-235, 2012. doi:10.7763/IJIMT.2012.V3.228 http://www.ijimt.org/show-37-455-1.html
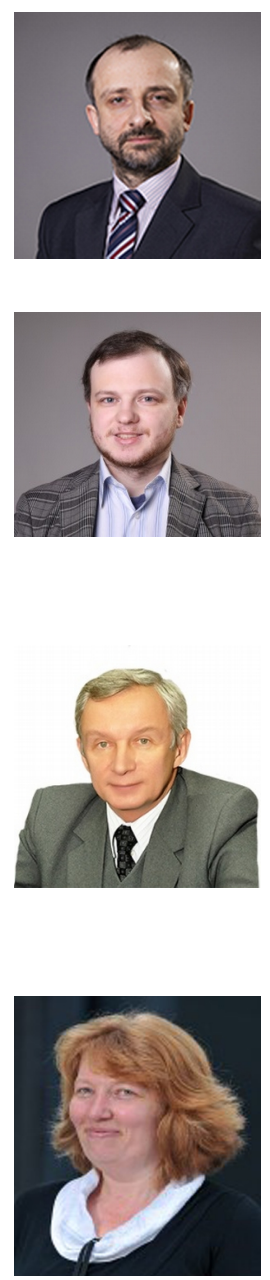

Rykhard Bohush Graduated from Polotsk State University in 1997. In 2002 he got his $\mathrm{PhD}$ in the field of Information Processing at the Institute of Engineering Cybernetics, the National Academy of Sciences of Belarus. Head of Computer Systems and Networks Department of Polotsk State University. His scientific interests include image and video processing, object representation and recognition, intelligent systems, digital steganography.

Pavel Yarashevich Born in 1991. In 2016 he got his the master degree in the field of Mathematical Modelling, Numerical Methods and Complexes of Programs. Currently he works as assistant of Computer Systems and Networks Department in PSU. Research interests are computer graphics processing, cryptography and programming.

Sergey Ablameyko DipMath in 1978, PhD in 1984, DSc in 1990, Prof. in 1992. Professor of Belarusian State University. His scientific interests are: image analysis, pattern recognition, digital geometry, knowledge based systems, geographical information systems, medical imaging. He is in Editorial Board of Pattern Recognition Letters, Pattern Recognition and Image Analysis and many other international and national journals. He is a senior member of IEEE, Fellow of IAPR, Fellow of Belarusian Engineering Academy, Academician of National Academy of Sciences of Belarus, Academician of the European Academy, and others. He was a First Vice-President of International Association for Pattern Recognition IAPR (2006-2008), President of Belarusian Association for Image Analysis and Recognition.

Tatiana Kalganova Graduated from Belarusian State University of Informatics and Radio-electronics. In 2000 she got his $\mathrm{PhD}$ in the field of Information Processing at the Napier University. Lecturer Brunel University London. The main research fields of Dr Kalganova are in Artificial Intelligence and its real-life applications: Evolutionary Design and Optimisation, Evolvable hardware, Modelling and optimisation of Large Systems, Operational research, Robotics, Swarm optimisation. 